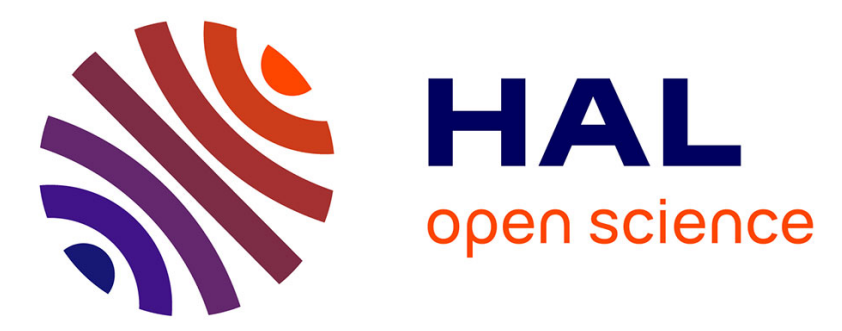

\title{
Incorporation de l'aluminium, du phosphore et du zinc dans les hétérojonctions AlxGa1-xPyAs 1-y-GaAs réalisées par épitaxie en phase liquide
}

\author{
L. Mayet, M. Gavand, A. Laugier
}

\section{- To cite this version:}

L. Mayet, M. Gavand, A. Laugier. Incorporation de l'aluminium, du phosphore et du zinc dans les hétérojonctions AlxGa1-xPyAs 1-y-GaAs réalisées par épitaxie en phase liquide. Revue de Physique Appliquée, 1979, 14 (1), pp.223-229. 10.1051/rphysap:01979001401022300 . jpa-00244580

HAL Id: jpa-00244580

https://hal.science/jpa-00244580

Submitted on 1 Jan 1979

HAL is a multi-disciplinary open access archive for the deposit and dissemination of scientific research documents, whether they are published or not. The documents may come from teaching and research institutions in France or abroad, or from public or private research centers.
L'archive ouverte pluridisciplinaire HAL, est destinée au dépôt et à la diffusion de documents scientifiques de niveau recherche, publiés ou non, émanant des établissements d'enseignement et de recherche français ou étrangers, des laboratoires publics ou privés. 


\title{
Incorporation de l'aluminium, du phosphore et du zinc dans les hétérojonctions $\mathrm{Al}_{x} \mathbf{G a}_{1-x} \mathbf{P}_{y} \mathbf{A s}_{1-y}$-GaAs réalisées par épitaxie en phase liquide
}

\author{
L. Mayet, M. Gavand et A. Laugier \\ Laboratoire de Physique de la Matière (*), I.N.S.A. de Lyon, \\ Bâtiment 502, 20 avenue A.-Einstein, 69621 Villeurbanne Cedex, France
}

(Reçu le 6 juillet 1978, révisé le 17 octobre 1978, accepté le 20 octobre 1978)

Résumé. - L'incorporation de phosphore $(<3,5 \%)$ dans la fenêtre $(\mathrm{Ga}, \mathrm{Al})$ As des photopiles $\mathrm{GaAs}$ conçues pour fonctionner sous concentration élevée permet d'annuler, dans la zone de température de fonctionnement, l'écart de paramètre cristallin et les contraintes mécaniques à l'interface. On montre que cette compensation peut être obtenue sans créer, pendant l'élaboration $(800,900 \mathrm{C})$, de nouveaux défauts. Des profils de concentration en $P$, $\mathrm{Al}, \mathrm{Zn}$ dans de simples hétérostructures réalisées par épitaxie liquide montrent que : 1) la concentration d'Al peut être maintenue constante ou bien peut créer un gradient de bande interdite favorable au rendement $\left.\left(10^{4} \mathrm{eV} \mathrm{m}^{-1}\right) ; 2\right)$ celle de $\mathrm{P}$ décroît de l'interface vers la surface $(6 \%$ par micron). Ceci est corrélé aux valeurs élevées du coefficient de partage déduit du diagramme de phase quaternaire ; 3 ) celle de l'accepteur $\mathrm{Zn}$ évolue de la même façon ; 4) le zinc diffuse dans le substrat sur quelques milliers d'angströms.

\begin{abstract}
The incorporation of small amounts of Phosphorus $(<3.5 \%)$ in the GaAlAs-GaAs heterostructure solar cells for high concentration can cancel the room temperature lattice mismatch and the stress at the interface. This is obtained without creation of new defects at the growth temperature $(900,800 \mathrm{C})$. Concentrations profiles of $\mathrm{P}, \mathrm{Al}, \mathrm{Zn}$ in single liquid phase epitaxy heterostructures indicate :

1) the $\mathrm{Al}$ composition may be constant. A graded band gap structure can also be obtained $\left(10^{4} \mathrm{eV} \mathrm{m}^{-1}\right)$;

2) Phosphorus concentration decreases from the interface to the surface $\left(6 \%\right.$ micron $\left.^{-1}\right)$ accordingly the quaternary phase diagram ;

3) the $\mathrm{Zn}$ concentration also decreases;

4) $\mathrm{Zn}$ diffuse into the substrate (a few thousands $\AA$ ).
\end{abstract}

1. Introduction. - Les photopiles en arséniure de gallium (GaAs) pourraient atteindre des rendements $n$ de $27 \%$ et fonctionner jusqu'à $300 \mathrm{C}$ [1]. De ce fait, elles sont particulièrement bien adaptées au fonctionnement sous concentration élevée. On peut même envisager la production simultanée d'électricité et de vapeur. Elles sont aussi intéressantes pour les applications où le coût (élevé) de ces cellules n'est pas un handicap majeur (applications spatiales par exemple). Actuellement, réalisées avec une couche superficielle en $\mathrm{Ga}_{1-x} \mathrm{Al}_{x} \mathrm{As}$ (fenêtre), ces photopiles détiennent le record du rendement avec ou sans concentration. Des valeurs $n=22 \%$ (sous illumination AM1) ont été obtenues [2] dépassant $24 \%$ sous 30 soleils. Sous 1700 soleils, des valeurs de n dépassant $19 \%$ ont été mesurées [3]. Le bon fonctionnement jusqu'à 5000 soleils a été rapporté [4]. Ces photopiles étaient réalisées par épitaxie en phase liquide (E.P.L.).

(*) Equipe de recherche associée au C.N.R.S.
Récemment, des photopiles élaborées en phase vapeur (décomposition des organométalliques) ont approché ces performances $[5,6]$. Pour les applications terrestres, le handicap majeur de ces dispositifs reste le prix de revient prohibitif. Le fonctionnement sous concentration élevée $(\simeq 1000)$ les rend largement compétitifs [7] à condition que leur durée de vie et leur fiabilité soient suffisantes. Les conditions de fonctionnement sont, en effet, particulièrement dures :

- densité de courant élevée (10 à $\left.20 \mathrm{~A} \mathrm{~cm}^{-2}\right)$

- température élevée (> $100 \mathrm{C}$ )

- irradiation intense

- cycles thermiques répétés, parfois brutaux.

On constate que, dans des conditions comparables, les diodes luminescentes et les lasers basés sur les mêmes matériaux se dégradent, parfois très rapidement. Certains mécanismes de dégradation font intervenir les contraintes au voisinage de l'interface dans ou près de la zone active. Ces contraintes sont 
dues à la différence de paramètres cristallins entre $\mathrm{GaAs}$ et $(\mathrm{Ga}, \mathrm{Al}) \mathrm{As}$. Cette différence est faible $(\Delta a / a=0,14 \%)$ pour le couple GaAs-AlAs, mais son influence néfaste a été reconnue [8]. Il a été proposé d'introduire du phosphore dans la fenêtre afin de compenser les contraintes [9]. Dans le cas des lasers à hétérostructure élaborés par E.P.L., Rozgonyi et al. [10] ont obtenu une amélioration des performances et de la durée de vie.

La structure optimale des cellules ( $\mathrm{Ga}, \mathrm{Al}) \mathrm{As}-\mathrm{GaAs}$ a été décrite en détail [1]. Typiquement, on a :

- la fenêtre en $(\mathrm{Ga}, \mathrm{Al}) \mathrm{As}$ avec $x>0,7$ pour bénéficier de la bande interdite indirecte, de type $\mathbf{P}\left(N_{\mathbf{A}}-N_{\mathbf{D}} \sim 10^{18} \mathrm{~cm}^{-3}\right.$, son épaisseur doit être inférieure à $0,5 \mu \mathrm{m})$;

- la zone $\mathrm{P}$ GaAs, d'épaisseur inférieure à $0,5 \mu$ $\left(N_{\mathrm{A}}-N_{\mathrm{D}} \sim 10^{19} \mathrm{~cm}^{-3}\right)$,

- la zone N GaAs $\left(N_{\mathrm{D}}-N_{\mathrm{A}} \sim 10^{17} \mathrm{~cm}^{-3}\right)$,

- le substrat $\mathrm{N} \mathrm{GaAs}\left(N_{\mathrm{D}}-N_{\mathrm{A}} \sim 10^{18} \mathrm{~cm}^{-3}\right)$.

Cette structure peut être réalisée par une triple épitaxie. Une simple hétérostructure peut suffire cependant, comme Woodall et Hovel [11] l'ont indiqué. La jonction p-n dans le substrat $\operatorname{GaAs}(N)$ était obtenue au cours de la croissance (E.P.L.) par diffusion du zinc à partir du bain.

Dans cet article, nous nous sommes limités à l'étude de l'incorporation d'Al, P, $\mathrm{Zn}$ dans la fenêtre (et éventuellement dans le substrat). Pour cela, nous avons réalisé par E.P.L. de simples hétérostructures $\mathrm{Al}_{x} \mathrm{Ga}_{1-x} \mathbf{P}_{y} \mathrm{As}_{1-y}$ sur substrat GaAs. On se propose de préciser les conditions de réduction de l'écart paramétrique et des contraintes par l'incorporation du phosphore.

2. Epitaxie en phase liquide. - La composition, $y$, du solide en phosphore, nécessaire pour réaliser la compensation des contraintes ainsi que le diagramme de phase quaternaire Ga-Al-As-P ont été calculés dans un précédent travail [9]. En raison des faibles valeurs de $y$ (inférieures à 0,04 ), ce dernier est très proche du diagramme ternaire Ga-Al-As. Les fractions molaires $x_{\mathrm{p}}^{l}$ de phosphore dans le liquide sont de l'ordre de $10^{-4}, 10^{-5}$. La figure 1 représente les variations des coefficients de distribution $K_{\mathrm{Al}}$, $K_{\mathrm{As}}, K_{\mathrm{P}}$ de l'aluminium, de l'arsenic et du phosphore en fonction de la température pour différentes valeurs de $x$, composition en aluminium du solide. On peut alors prévoir les profils en aluminium et en phosphore dans la couche. Si $K_{\mathrm{Al}}>K_{\mathrm{As}}$, le bain s'appauvrit en aluminium et $x$ décroît, de l'interface vers la surface du dépôt. C'est l'inverse si $K_{\mathrm{Al}}<K_{\mathrm{As}}$. La ligne en trait plein représente le lieu des points pour lesquels $K_{\mathrm{As}}=K_{\mathrm{Al} \mathrm{l}}$. Alors, $x$ peut être maintenu constant. Nous avons choisi de travailler avec des profils plats en aluminium, à deux températures $800 \mathrm{C}$ et $900 \mathrm{C}$, ceci afin de réduire les effets de matrice dans la détermination des profils de $\mathbf{P}$ et $\mathrm{Zn}$ et pour simplifier l'étude de la compensation des contraintes. Ces

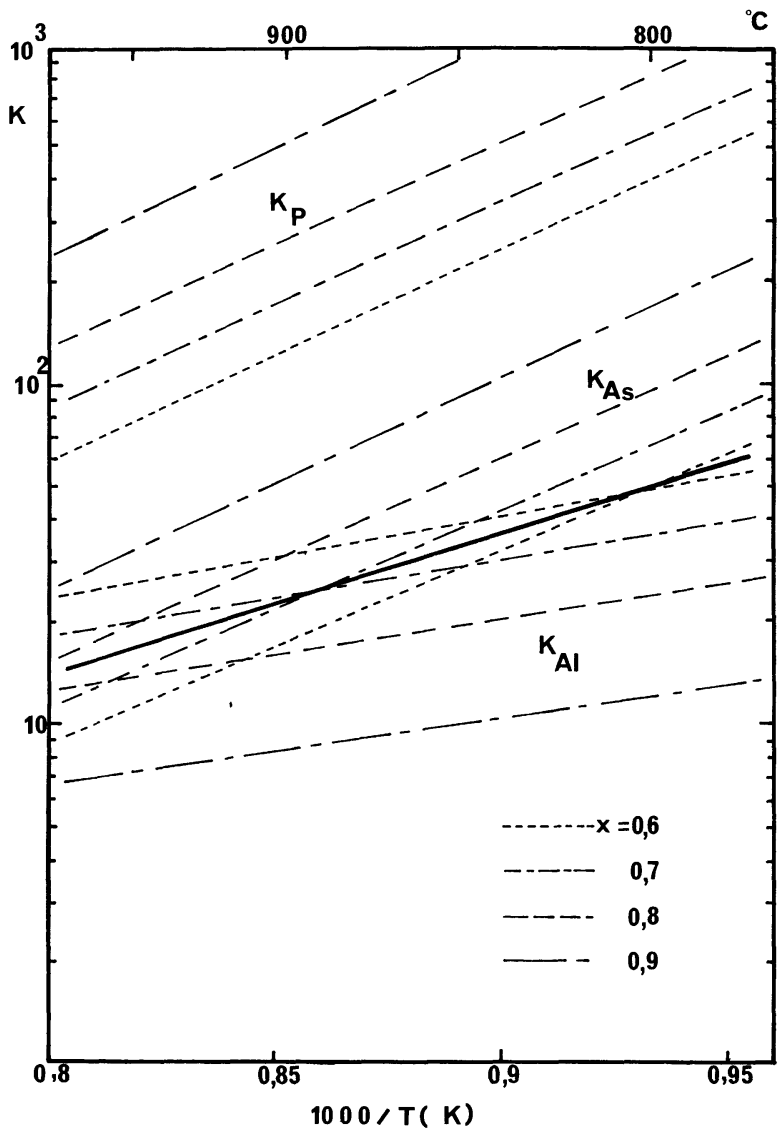

Fig. 1. - Coefficients de distribution de l'aluminium, de l'arsenic et du phosphore, $K_{\mathrm{AL}}, K_{\mathrm{As}}, K_{\mathrm{P}}$, en fonction de la température pour différentes valeurs de la concentration en aluminium du solide $x$. La ligne en trait plein représente les points où $K_{\mathrm{As}}=K_{\mathrm{Al}}$.

[Distribution coefficients of aluminium, arsenic and phosphorus $K_{\mathrm{Al}}, K_{\mathrm{As}}, K_{\mathrm{p}}$ versus temperature for several values of aluminium concentration in the solid. The full line gives $K_{\mathrm{As}}=K_{\mathrm{AL}}$.]

conditions mènent à des valeurs de $x$ respectivement égales à 0,61 et 0,71 . On a alors à $800 \mathrm{C}$ :

$K_{\mathrm{As}}=K_{\mathrm{A} 1}=50$ et $K_{\mathrm{P}}=410$.

A $900 \mathrm{C}$, ces valeurs deviennent respectivement 23 et 180 .

Les dépôts ont été réalisés dans un four vertical à système rotatif décrit par ailleurs $[9,12]$. Les quantités prévues par le diagramme de phase sont pesées avec soin. On a typiquement pour $800 \mathrm{C}: \mathrm{Ga}=2 \mathrm{~g}$, $\mathrm{Al}=4,8 \mathrm{mg}, \mathrm{GaP}=100 \mu \mathrm{g}, \mathrm{Zn}=5 \mathrm{mg}$; pour $900 \mathrm{C}: \mathrm{Ga}=2 \mathrm{~g}, \mathrm{Al}=11,7 \mathrm{mg}, \mathrm{GaP}=210 \mu \mathrm{g}$, $\mathrm{Zn}=12 \mathrm{mg}$. Le mélange $\mathrm{Ga}, \mathrm{P}, \mathrm{Al}, \mathrm{Zn}$ est saturé en GaAs à la température de début de croissance. La température est alors abaissée de $10 \mathrm{C}$ à la vitesse de $0,25 \mathrm{C} / \mathrm{mn}$. Les épaisseurs moyennes obtenues sont typiquement $4 \mu \mathrm{m}$ à $800 \mathrm{C}$ et $10 \mu \mathrm{m}$ à $900 \mathrm{C}$. Ces épaisseurs sont trop importantes pour la réalisation de bonnes fenêtres en raison de l'absorption optique mais permettent une étude plus aisée des profils et des contraintes. 
Des profils en $x$ ont été obtenus à la microsonde électronique sur tranche polie ou clivée. La figure 2 montre trois profils effectués sur des dépôts réalisés à $900 \mathrm{C}$. Soit $x_{\mathbf{i}}$ la concentration en aluminium de la première couche au voisinage du substrat. Conformément aux prévisions précédentes, on observe bien que pour $x_{\mathbf{i}}<0,7$ la composition en aluminium décroît à partir de l'interface alors qu'elle croît pour $x_{\mathbf{i}}>0,7$. Dans ce cas, l'effet d'élargissement de la bande interdite vers la surface du dépôt crée un champ électrique de l'ordre de $10^{4} \mathrm{~V} \mathrm{~m}^{-1}$ qui, en favorisant le déplacement des porteurs vers la jonction, permet d'améliorer le rendement [13, 14].

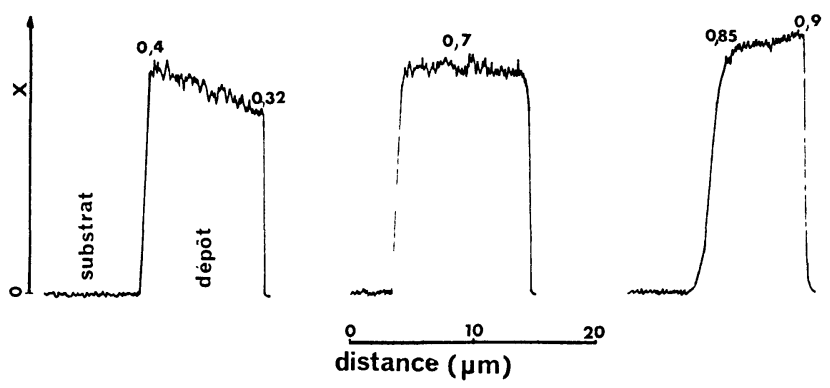

Fig. 2. - Profils à la microsonde électronique de la concentration en aluminium de dépôts ternaires réalisés à $900 \mathrm{C}$.

[Electron microprobe analysis of aluminium concentration across ternary films grown at $900 \mathrm{C}$.]

3. Caractérisation des couches. - 3.1 Profils EN PHOSPHORE ET EN ZINC. - Les valeurs de $K_{\mathrm{P}}$ sont très élevées. Ce coefficient augmente avec $x$ et lorsque la température diminue. La figure 1 montre qu'il n'est pas possible d'obtenir $K_{\mathrm{P}}=K_{\mathrm{As}}=K_{\mathrm{A} 1}$. Il y aura donc une diminution de la concentration en phosphore à travers la couche, d'autant plus que la température est abaissée pendant la croissance. Des profils ont été effectués au microanalyseur ionique CAMECA pour $\mathrm{Al}, \mathrm{P}$ et $\mathrm{Zn}$ sur une couche $\mathrm{Ga}_{0,39} \mathrm{Al}_{0,61} \mathrm{P}_{y} \mathrm{As}_{1-y}$ dopée au zinc et réalisée à $800 \mathrm{C}$ (Fig. 3). La valeur moyenne de $y$ est estimée à 0,013 à partir de mesures de diffraction X, comme indiqué plus loin. L'épaisseur mesurée au microscope interférentiel est 3,85 $\mu \mathrm{m}$. Jusqu'à l'interface, la vitesse de pulvérisation était de $4,5 \AA / \mathrm{s}$. Dans GaAs, pour une même valeur du courant ionique primaire, elle est de l'ordre de $10 \AA / \mathrm{s}$. Le profil en aluminium étant plat, la vitesse d'érosion est constante dans toute l'épaisseur de la couche et ne varie qu'à l'interface. Ce dernier point rend délicat la détermination précise de la position de l'interface. Le diamètre du cratère étant de $2 \mathrm{~mm}$, les effets de bords étaient éliminés en limitant la collection sur la portion centrale par un diaphragme de $50 \mu \mathrm{m}$ de diamètre.

La diminution de la concentration en phosphore, à partir de l'interface, est d'environ $6 \%$ par micron. Ceci est en accord avec le diagramme de phase et

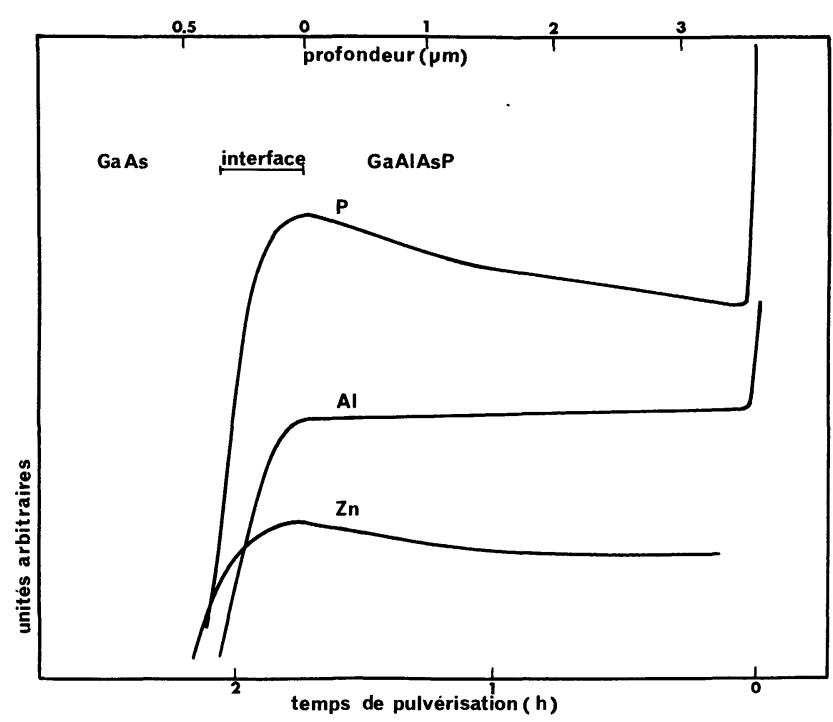

Fig. 3. - Profils de composition en phosphore, zinc et aluminium obtenus par microanalyse ionique sur un dépôt $\mathrm{Ga}_{0,39} \mathrm{Al}_{0,61} \mathrm{As}_{1-y} \mathrm{P}_{\boldsymbol{y}}$.

[Composition profiles of phosphorus, zinc and aluminium given by ionic microanalysis on $\mathrm{Ga}_{0.39} \mathrm{Al}_{0.61} \mathrm{As}_{1-y} \mathrm{P}_{y}$ film.]

comparable aux résultats obtenus par Chang et al. [15] en spectroscopie Auger pour des couches

$$
\mathrm{Ga}_{0,64} \mathrm{Al}_{0,36} \mathrm{P}_{y} \mathrm{As}_{1-y}
$$

avec $y$ voisin de 0,01 . Le profil de concentration en zinc est relativement plat. A partir des mesures capacitives, la concentration moyenne est estimée à $3 \times 10^{17} \mathrm{~cm}^{-3}$.

Dans la zone de l'interface, la diminution de la concentration en $\mathrm{Al}, \mathrm{P}$ et $\mathrm{Zn}$ peut laisser supposer une diffusion de tous ces éléments sur quelques milliers d'angströms dans GaAs. Ce n'est pas le cas. Le coefficient de diffusion $D$ d'Al dans $\mathrm{GaAs}$ à $800 \mathrm{C}$ est de l'ordre de $10^{-18} \mathrm{~cm}^{2} / \mathrm{s}$ [16]. Dans les conditions de croissance utilisées $(t=40 \mathrm{mn})$, la profondeur de diffusion ne dépasse pas quelques angströms et on peut écarter l'hypothèse d'une dissolution préalable du substrat. On est donc amené à supposer que cette diminution est due, comme cela est souvent observé, à un défaut de parallélisme entre le fond du cratère et l'interface vrai (angle lapping). Dans ces conditions, on peut aussi conclure que, comme $\mathrm{Al}, \mathrm{P}$ diffuse peu dans le substrat.

Il n'en est pas de même pour le zinc. La profondeur de diffusion estimée à partir de la figure 3 est de $2000 \AA$. Le calcul de la profondeur de diffusion de $\mathrm{Zn}$ est compliqué par le fait que $D_{\mathrm{Zn}}$ dépend de la concentration superficielle $N_{\mathrm{Zn}}$ à température donnée. Weissberg [17] indique que $D$ est proportionnel à $N_{\mathbf{Z n}_{\mathbf{n}}}^{2}$. A $800 \mathrm{C}$, on obtient $D_{\mathrm{Zn}}=10^{-14} \mathrm{~cm}^{2} / \mathrm{s}$ pour $N_{\mathrm{Zn}}=3 \times 10^{17} \mathrm{~cm}^{-3}$ à partir des valeurs données par Casey [18] pour $N_{\mathrm{Zn}}=1,6 \times 10^{20} \mathrm{~cm}^{-3}$. On calcule alors une longueur de diffusion de $1000 \AA$, 
compatible avec le profil obtenu. Ainsi, le substrat étant dopé avec $2 \times 10^{17} \mathrm{~cm}^{-3}$ donneurs $(\mathrm{Si})$, la jonction se trouve localisée à moins de $2000 \AA$ de l'interface.

On observe aussi, sur la figure 3, une remontée brutale en $\mathrm{Al}$ et $\mathrm{P}$ à la surface. Un tel phénomène est très souvent observé $[19,20]$. Il n'est pas expliqué dans notre cas, mais il ne semble pas être un effet de mesure [21]. L'accroissement correspondant en AlP pourrait être corrélé avec la résistance série qui pose un problème dans ce type de photopile. Ces points restent à approfondir.

3.2 BANDES INTERDITES. - Dans la structure photovoltaïque considérée, la couche superficielle doit avoir une structure de bande indirecte. Sa largeur $E_{\mathbf{g}_{i}}$ n'est pas aisément mesurable directement. Par contre, la largeur de la bande interdite directe $E_{\mathrm{gd}}$ peut être obtenue simplement et avec précision (quelques $\mathrm{meV}$ à $300 \mathrm{~K}$ ) à partir des spectres de réflectivité. Nous avons utilisé la méthode de réflectivité différentielle par modulation de longueur d'onde. Cette technique est rapide et non destructive (pas de préparation spéciale, pas de contact). L'épaisseur analysée est inférieure à $0,5 \mu \mathrm{m}$. La figure 4 représente un spectre typique. En outre, il est possible d'avoir des informations sur le parallélisme surface-interface et sur la qualité de l'interface. Pour des énergies inférieures au gap, la réflexion sur cet interface peut produire un phénomène d'interférence. A partir des ondulations correspondantes, on déduit l'épaisseur de la couche. Dans le cas de la figure 4, relative à un ternaire, en prenant 3,3 pour la valeur de l'indice de réfraction, on obtient une épaisseur de $4,85 \pm 0,1 \mu \mathrm{m}$. La valeur de $E_{\mathrm{g}}$ est de $2,43 \mathrm{eV}$. Comme on va le discuter, cette valeur permet d'obtenir une estimation de $x$, ici $x=0,72$.

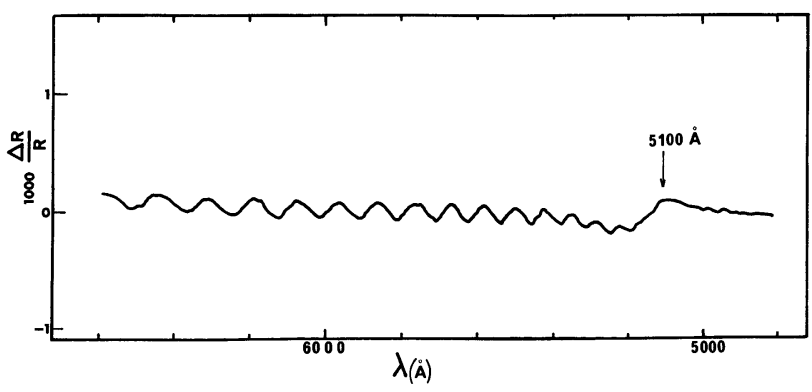

Fig. 4. - Spectre de modulation de longueur d'onde obtenu sur un dépôt $\mathrm{Ga}_{1-x} \mathrm{Al}_{x} \mathrm{As}$ réalisé à $900 \mathrm{C}$. L'épaisseur du dépôt est $4,85 \pm 0,1 \mu$. Le gap direct $2,43 \mathrm{eV}$ donne $x=0,72$.

[Spectral response of wavelength modulation analysis on $\mathrm{Ga}_{1-x} \mathrm{Al}_{x} \mathrm{As}$ film grown at $900 \mathrm{C}$. The thickness of the film is $4.85 \pm 0.1 \mu$. The value of direct bandgap $2.43 \mathrm{eV}$ gives $x=0.72$.]

La concentration superficielle en aluminium peut, en effet, être obtenue à partir de la relation :

$E_{\mathbf{g}_{\mathbf{d}}}(\mathrm{eV})=1,44+1,04 x+0,47 x^{2}+1,34 y$.
Cette relation approchée, reliant la largeur de la bande interdite directe du composé quaternaire aux compositions $x$ et $y$, est obtenue à partir des relations correspondantes dans le cas des ternaires $(\mathrm{Ga}, \mathrm{Al}) \mathrm{As}$ et $\mathrm{Ga}(\mathrm{As}, \mathrm{P})$ [22]. Le terme quadratique en $y$ a été négligé compte tenu des faibles teneurs en phosphore utilisées. Pour la même raison, nous avons aussi négligé le terme croisé en $x y$ caractéristique des alliages quaternaires (qui n'est d'ailleurs pas très bien connu) [23]. De plus, en raison des variations de $x$ et $y$ au voisinage de la surface, il serait illusoire d'espérer sur $x$ une précision relative meilleure que quelques pour cent. Ces restrictions étant faites, cette méthode se révèle être très intéressante en raison de sa commodité et de sa rapidité. Enfin, pour conclure, il faut signaler que, $x$ et $y$ étant connus, la valeur de la bande interdite peut être estimée de deux façons :

- à l'aide de la relation approchée suivante, obtenue de la même façon que la relation (1) [9] :

$E_{\mathbf{g}_{\mathbf{i}}}(\mathrm{eV})=1,92+0,23 x+0,34 y+0,01 x y$

- en se référant aux abaques de la référence [23] obtenues par une méthode d'interpolation plus sophistiquée.

4. Compensation des contraintes à l'interface. 4.1 Mesure Des CONTRAINTES. - A la température de croissance $(800 \mathrm{C})$, le désaccord paramétrique entre un cristal de GaAs et un cristal de AlAs est faible. Il est nul à $850 \mathrm{C}$. Il en est de même pour un cristal mixte ternaire $\mathrm{Ga}_{1-x} \mathrm{Al}_{x} \mathrm{As}$. Mais, par suite des coefficients de dilations linéaires différents de ces matériaux : GaAs : $\alpha=6,86 \times 10^{-6} \mathrm{C}^{-1}$ [24], AlAs : $\alpha=5,2 \times 10^{-6} \mathrm{C}^{-1}$ [25], un écart apparaît lors du retour à la température ambiante. Cet écart est relativement important : $1,4 \times 10^{-3}$ pour AlAs et $8,5 \times 10^{-4}$ pour $\mathrm{Ga}_{0,39} \mathrm{Al}_{0,61}$ As (Fig. 5), l'adaptation des réseaux se fait par l'apparition d'une contrainte

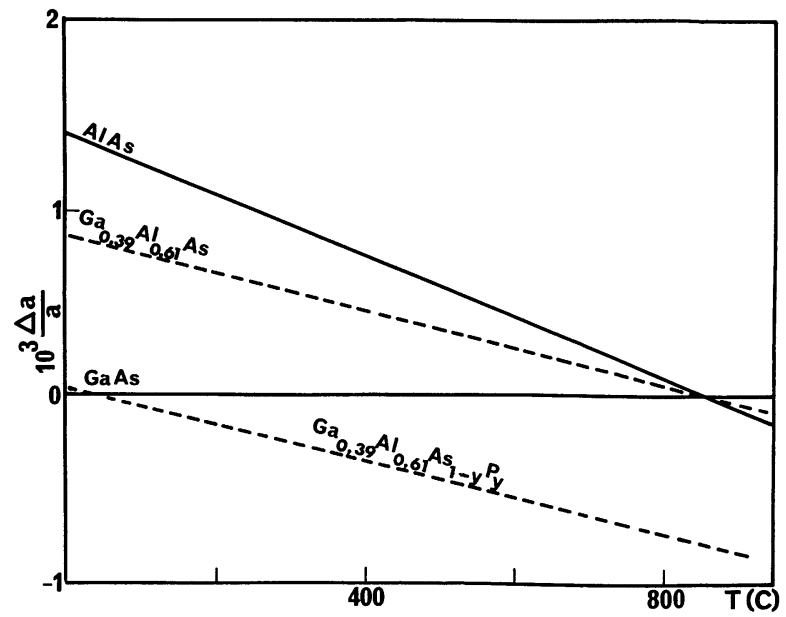

Fig. 5. - Ecart paramétrique relatif avec le $\mathrm{GaAs}$ en fonction de la température pour AlAs, $\mathrm{Ga}_{0,39} \mathrm{Al}_{0,61} \mathrm{As}$ et $\mathrm{Ga}_{0,39} \mathrm{Al}_{0,61} \mathrm{As}_{1-y} \mathrm{P}_{\mathrm{y}}$.

[Relative lattice parameter mismatch from GaAs versus temperature for $\mathrm{AlAs}, \mathrm{Ga}_{0.39} \mathrm{Al}_{0.61} \mathrm{As}$ and $\mathrm{Ga}_{0.39} \mathrm{Al}_{0.61} \mathrm{As}_{1-y} \mathrm{P}_{y}$.] 
élastique dans le matériau. Cette contrainte peut être réduite par l'incorporation de phosphore. Selon la quantité $y$ de phosphore introduite, le quaternaire permet d'accorder les paramètres cristallins à la température de fonctionnement du dispositif. La détermination de $y$ se fait au goniomètre à rayons $\mathrm{X}$ par la mesure à $25 \mathrm{C}$ de l'écart $\Delta d$ entre la distance interréticulaire d'un plan cristallographique du film et celle du substrat. Le dispositif étant contraint, cet écart ne représente pas l'écart normal entre deux cristaux libres. Une normalisation est donc nécessaire en utilisant l'écart maximum correspondant à $y=0$ et en supposant la loi de Végard vérifiée.

La figure 6 montre l'évolution du spectre de la réflexion $K_{\alpha 1}$ du cuivre sur le plan 444. Le pic satellite $\mathrm{GaAlAsP}$ se déplace relativement vers la gauche du pic GaAs à mesure que la quantité de phosphore dans le bain augmente. Pour la valeur la plus importante de $y$, le paramètre du quaternaire devient inférieur à celui de GaAs. Il existe ainsi une valeur de $y$ pour laquelle l'accord de parạmètre est réalisé. Avec $x=0,61$ et $y=0,026$, les paramètres sont accordés

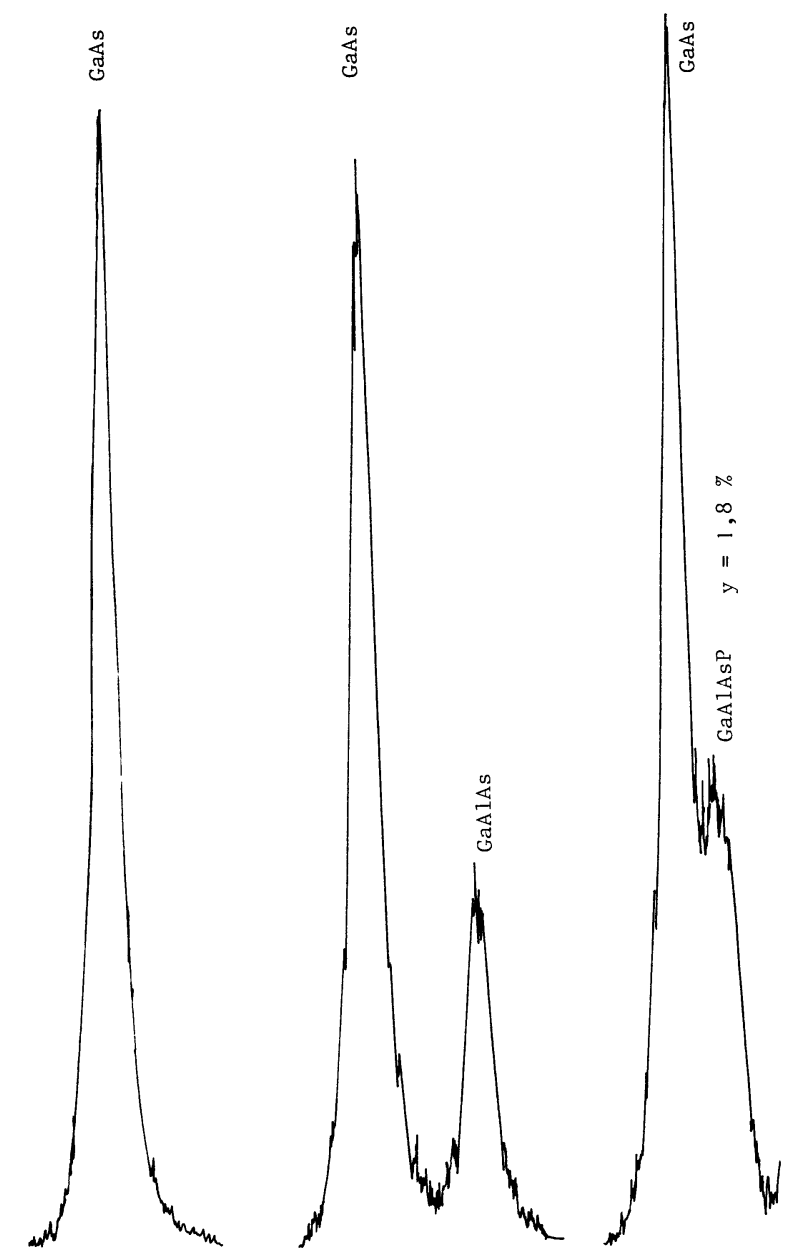

Fig. 6. - Influence de l'incorporation de phosphore sur l'écart paramétrique. Réflexion $\mathrm{CuK} \alpha_{1} 444$ simple cristal $x=0,61$, $T_{\mathrm{c}}=800 \mathrm{C}$. Le pic $\mathrm{Ga}_{1-x} \mathrm{Al}_{x} \mathrm{As}_{1-y} \mathrm{P}_{y}$ se déplace vers les grands angles lorsque $y$ augmente. Pour $y=2,6 \%$, l'écart est pratiquement annulé. à $25 \mathrm{C}$. Cette valeur de $y$ est, en fait, une moyenne à $\pm 10 \%$ sur le profil de concentration dans toute l'épaisseur de la couche.

4.2 Limitation A LA RÉDUCTION DES CONTRAINTES. - Il est évident que si on augmente encore la quantité de phosphore dans la couche, les contraintes à la température de croissance deviennent excessives et un réseau de dislocations se crée alors. On a observé (Fig. 7a) l'apparition de ce réseau bidimensionnel de dislocations de désaccord à l'interface sur une couche d'épaisseur $e=4 \mu \mathrm{m}$ obtenue à $800 \mathrm{C}$ avec $x=0,61$ et $y=0,031$. Celui-ci a été observé par d'autres auteurs sur Ge-GaAs [26], $\mathrm{Ga}(\mathrm{As}, \mathrm{P})-\mathrm{GaAs}$ [27] et surtout $\mathrm{Ga}_{1-x} \mathrm{Al}_{x} \mathrm{As}_{1-y} \mathrm{P}_{y}$-GaAs [28, 29] avec $x$ compris entre 0,2 et 0,36 . Dans ce dernier cas, il a été récemment confirmé [29] que ce réseau était essentiellement constitué de dislocations $\langle 110\rangle$ de type $60^{\circ}$.

Le mécanisme le plus probable concernant l'apparition de ce réseau est celui proposé par Matthews et al. [30] : les dislocations de désaccord à l'interface proviennent des dislocations du substrat soumises à

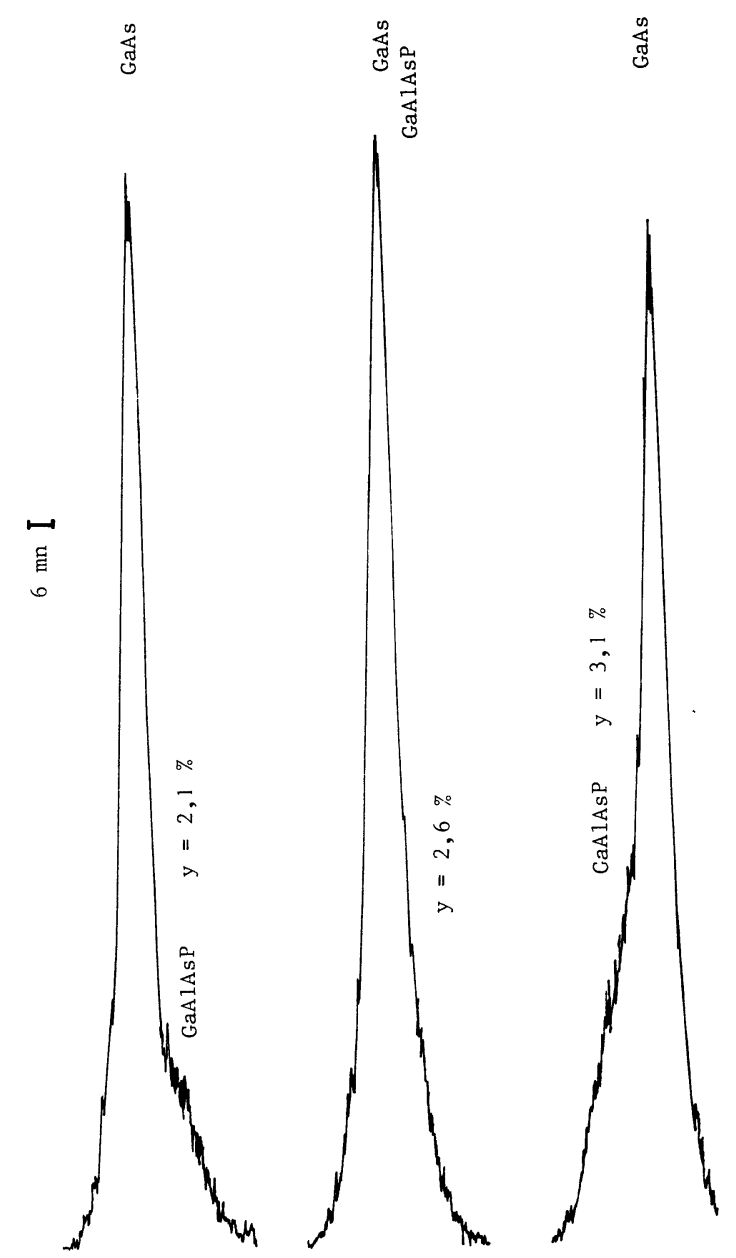

[Influence of phosphorus incorporation on lattice mismatch. Reflection $\operatorname{CuK} \alpha_{1} 444$, single crystal $x=0.61, T_{\mathrm{c}}=800 \mathrm{C}$. The $\mathrm{Ga}_{1-x} \mathrm{Al}_{x} \mathrm{As}_{1-y} \mathrm{P}_{y}$ peak shifts on the left when $y$ increases. For $y=2.6 \%$ the matching is nearly perfect.] 


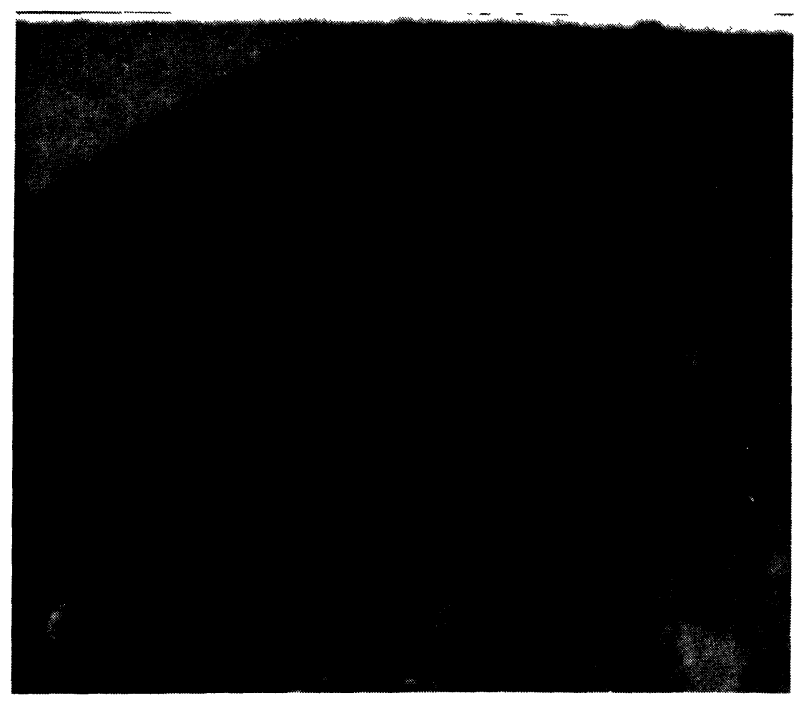

a)

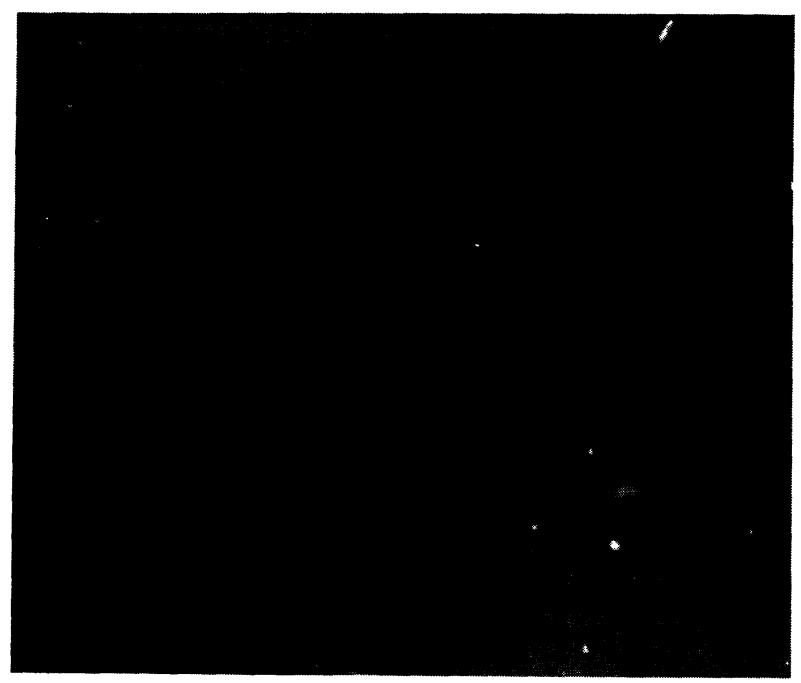

b)

Fig. 7. - Topographies Berg-Barrett réflexion

$440 \mathrm{Ga}_{0,39} \mathrm{Al}_{0,61} \mathrm{As}_{1-y} \mathrm{P}_{y} / \mathrm{GaAs}$

a) $y=0,031 ; e=4 \mu$; réseau de dislocations orientées $\langle 110\rangle$ b) $y=0,013 ; e=3 \mu$.

[Berg Barett topographs (440). Reflection on $\mathrm{Ga}_{0.39} \mathrm{Al}_{0.61} \mathrm{As}_{1-y} \mathbf{P}_{y} /$ GaAs (111) structure. a) $y=0.031 ; e=4 \mu$; the dislocation array is oriented in the direction $\langle 110\rangle b) y=0.013$; $e=3 \mu$.]

une force de contrainte de tension. Celle-ci peut les faire glisser à l'interface lorsqu'un certain seuil de tension, fonction de l'épaisseur de la couche, est dépassé. L'épaisseur critique, en fonction de $x_{\mathrm{p}}$ en dessous de laquelle les contraintes ne sont pas relâchées plastiquement à $790 \mathrm{C}$ a été déterminée expérimentalement par Rozgonyi et al. [31] pour $x=0,34 . K_{\mathrm{p}}$ a aussi été mesuré : $K_{\mathrm{p}}=290$ [15]. Utilisant cette valeur, nous avons reporté (Fig. 8) cette courbe de seuil sur le diagramme $y=f$ (épaisseur). Cette représentation est préférée ici à celle de la référence [31]

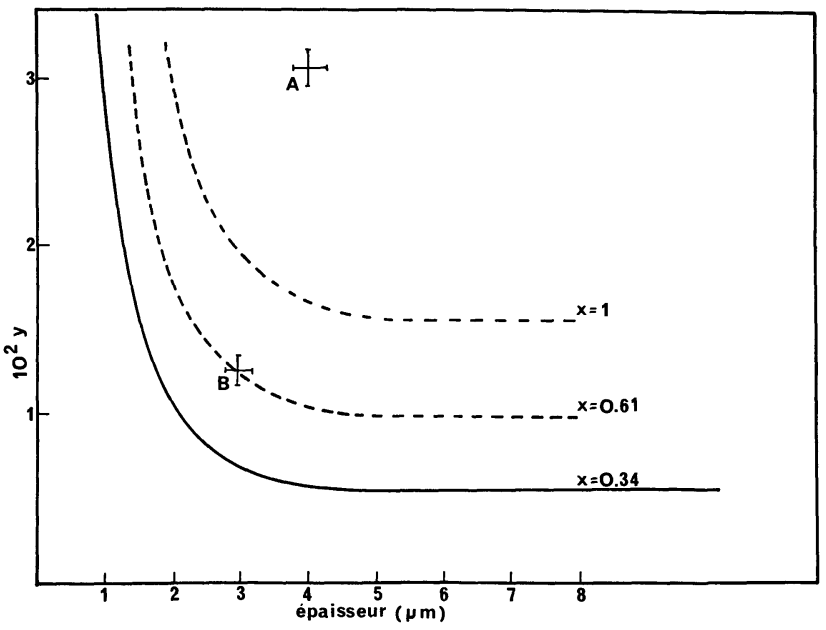

Fig. 8. - Concentration en phosphore $y$ et épaisseur critique des couches quaternaires déposées à $800 \mathrm{C}$ sur GaAs. Les courbes pour $x=1$ et 0,61 sont déduites, comme expliqué dans le texte, de la courbe $x=0,34$ obtenue à partir des résultats de Rozgonyi et al. [31]. Les points expérimentaux A et $\mathrm{B}$ correspondent aux figures $7 a$ et $7 b$ respectivement.

[Phosphorus concentration and critical thickness of quaternary layers grown on $\mathrm{GaAs}$ at $800 \mathrm{C}$. The curve for $x=0.34$ is deduced from the data of Rozgonyi and al. [31]. Points A and B correspond to layers of figures $7 a$ and $7 b$ respectively.]

pour faciliter les comparaisons avec d'autres valeurs de $x$ sans faire intervenir le diagramme de phase. On constate que, pour les faibles valeurs de $y$, il n'existe pas d'épaisseur critique, ce qui s'explique de la façon suivante. Pour le ternaire, le désaccord paramétrique qui serait nul vers $850 \mathrm{C}$ conduit, à $790 \mathrm{C}$, à des contraintes élastiques de compression. Pour $y=0,54 \%$, ces contraintes sont annulées. Au cours $\mathrm{du}$ refroidissement, les couches avec $y \leqslant 0,54 \%$ subissent des contraintes croissantes, mais celles-ci demeurent des contraintes de compression. Quelle que soit l'épaisseur, on reste dans le domaine élastique sans apparition de dislocations. Au contraire, pour les valeurs supérieures de $y$, on pénètre (à $790 \mathrm{C}$ ) dans le domaine des contraintes de tension. Comme Matthews et al. l'ont montré, l'épaisseur joue un rôle critique [30]. Pour annuler les contraintes au voisinage de la température ambiante, il faut travailler cependant dans ce domaine. Il est donc impératif de limiter l'épaisseur de la couche déposée si on veut éviter l'apparition d'un réseau de dislocation à l'interface, c'est-à-dire près de la zone active de la photopile. La figure $7 b$ montre la topographie d'un tel dépôt $(y=0,013 ; x=0,61 ; e=3 \mu \mathrm{m} ; T=800 \mathrm{C})$. En se reportant à la figure 8 , on constate que les points correspondant aux deux dépôts $(7 a, 7 b)$ sont tous les deux situés au-dessus de la courbe de Rozgonyi et al., donc dans la zone d'apparition du réseau de dislocations ce qui n'est pas observé pour $7 b$. Ceci est normal car il convient d'adapter la courbe de seuil à nos conditions expérimentales : $T=800 \mathrm{C}$ et $x=0,61$. Les températures sont assez voisines pour qu'on puisse 
les confondre. Par contre, il faut tenir compte de $x$. En première approximation, la courbe de seuil a donc simplement été décalée vers le haut, dans le rapport $61 / 34$ et reportée figure 8 . Le point correspondant à $7 b$ est situé au voisinage immédiat de cette courbe. Pour $7 a$, il est encore nettement au-dessus. Compte tenu de l'imprécision existant sur la courbe de base et des hypothèses implicites de notre raisonnement, l'accord est bon.

4.3 CAS DES PHOtopiles. - On se trouve alors dans une situation plus favorable que dans le présent travail, pour deux raisons. D'abord, on travaille avec $x>0,7$; de ce fait, la courbe de seuil se trouvera située encore plus haut. Sur la figure 8 , nous avons rajouté la courbe (supposée) pour $x=1$. Ensuite, la réduction de l'absorption optique dans la fenêtre impose de limiter son épaisseur à moins de $0,5 \mu \mathrm{m}$. Or, nous pouvons estimer l'épaisseur critique maximum. A $25 \mathrm{C}$, le plus grand écart $\Delta a / a$ qu'il faut compenser est de $0,14 \%$ pour $x=1$. Il faut alors $y=0,034$. En se reportant figure 8 on trouve une épaisseur critique, en tout cas, supérieure au micron. L'incorporation de phosphore maintiendra donc la couche déposée dans ses limites élastiques.

5. Conclusion. - Ainsi, la compensation des contraintes peut être réalisée dans la zone de température de fonctionnement envisagée pour les photopiles sous concentration élevée. Afin de conclure définitivement sur l'intérêt de cette compensation, il est nécessaire, à présent, d'entreprendre une étude systématique des centres profonds dans et au voisinage de la zone active et de procéder à des études de vieillissement, comme il a été fait récemment pour les diodes électroluminescentes [32].

Remerciements. - Ce travail a été soutenu par l'A.T.P.-C.N.R.S. Conversion Photovoltaïque. Nous remercions aussi C. Henry (Thomson-C.S.F.) ainsi que B. Blanchard (C.E.N.G.) pour leur aide matérielle.

\section{Bibliographie}

[1] Hovel, H. J., Solar Cells, vol. 11 of Semiconductors and Semimetals. R. K. Willardson and A. C. Beer eds. (Academic Press, N.Y.) 1976.

[2] Woodall, J. M., Hovel, H. J., Appl. Phys. Lett. 30 (1977) 492.

[3] James, L. W., Moon, R. L., Conf. Rec. 11th I.E.E. Photovoltaic Specialist Conference Scottsdale (1975) 402.

[4] Davis, R., KNIGHT, J. R., Solar Energy 17 (1975) 145.

[5] Fabre, E., Briere, A., Andre, J. P., Photovoltaic Solar Energy Conference (Luxembourg) 1977, 388.

[6] Nelson, N. J., Johnson, K. K., Moon, R. L., van Der Plas, H. A., James, L. W., Appl. Phys. Lett. 33 (1978) 26.

[7] Hovel, H. J., I.B.M. J. Res. Dev. 22 (1978) 112.

[8] Dyment, J. C., Nash, F. R., Hwang, C. J., Rozgonyi, G. A., Hartman, R. L., Marcos, H. H., Maszko, S. E., Appl. Phys. Lett. 24 (1974) 481.

[9] Laugier, A., Mayet, L., Gavand, M., Photovoltaic Solar Energy Conference, Luxembourg (1977) 1080

[10] Rozgonyi, G. A., Panish, M. B., Appl. Phys. Lett. 23 (1973) 533.

[11] Woodall, J. M., Hovel, H. J., Appl. Phys. Lett. 21 (1972) 379.

[12] Mayet, L., Montegu, B., Gavand, M., J. Cryst. Growth 39 (1977) 362.

[13] Marfaing, Y., Proc. Int. Coll. Solar Cells (Gordon and Breach) 1970,67

[14] Hutchiy, J. A., Appl. Phys. Lett. 26 (1975) 457.

[15] Chang, C. C., Panish, M. B., Wagner, W. R., Rode, D. L., Sumski, S., Sobers, R. G., J. Appl. Phys. 47 (1976) 3752.
[16] Chang, L. L., Koma, A., Appl. Phys. Lett. 29 (1976) 138.

[17] Weisberg, L. R., Trans. Met. Soc. AIME 230 (1964) 291.

[18] Casey, H. C., Panish, M. B., Trans. Met. Soc. AIME 242 (1968) 406.

[19] Gauneau, A. M., Analysis 5 (1977) 357.

[20] Blanchard, B., Pittsburg Int. Conf. 1973.

[21] Blanchard, B., Communication privée.

[22] Onton, A., Lorentz, M. R., Woodall, J. M., ChicoKRA, R. C., J. Cryst. Growth 27 (1974) 166.

[23] Glisson, T. H., Hauser, J. R., Littlejohn, M. A. WilLiAMs, C. K., J. Electron. Mat. 7 (1978) 1.

[24] Pierron, E. D., Parker, D. L., McNeely, J. B., J. Appl. Phys. 38 (1967) 4669.

[25] Ettenberg, M., Paff, R. J., J. Appl. Phys. 41 (1970) 3926.

[26] Meieran, E. S., J. Electrochem. Soc. 114 (1967) 292.

[27] Matthews, J. W., Blakeslee, A. E., J. Cryst. Growth 27 (1974) 118.

[28] Rozgonyi, G. A., Petroff, P. M., Panish, M. B., Appl. Phys. Lett. 24 (1974) 251.

[29] Petroff, J. F., Sauvage, M., J. Cryst. Growth 43 (1978) 628.

[30] Matthews, J. W., Mader, S., Light, T. B., J. Appl. Phys. 41 (1970) 3800.

[31] Rozgonyi, G. A., Petroff, P. M., Panish, M. B., J. Cryst. Growth 27 (1974) 106.

[32] Balland, B., Revue Phys. Appl. 13 (1978) 232. 